Acta Crystallographica Section E

\section{Structure Reports}

Online

ISSN 1600-5368

\section{H. S. Yathirajan, ${ }^{a}$ Anil N.} Mayekar, ${ }^{a}$ B. Narayana, ${ }^{\text {b }}$ B. K. Sarojini $^{c}$ and Michael Bolte ${ }^{d_{*}}$

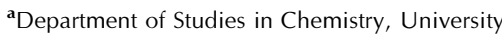
of Mysore, Manasagangotri, Mysore 570006 , India, 'bepartment of Chemistry, Mangalore University, Mangalagangotri 574 199, India, ${ }^{\mathrm{c}}$ Department of Chemistry, P. A. College of Engineering, Nadupadavu, Mangalagangotri 574 153, India, and Institut für Anorganische Chemie, J. W. Goethe-Universität Frankfurt, Max-von-Laue-Strasse 7, 60438 Frankfurt/Main, Germany

Correspondence e-mail:

bolte@chemie.uni-frankfurt.de

\section{Key indicators}

Single-crystal X-ray study

$T=173 \mathrm{~K}$

Mean $\sigma(\mathrm{C}-\mathrm{C})=0.002 \AA$

$R$ factor $=0.034$

$w R$ factor $=0.090$

Data-to-parameter ratio $=14.4$

For details of how these key indicators were automatically derived from the article, see http://journals.iucr.org/e.

\title{
(2E)-1-(2,4-Dichlorophenyl)-3-(6-methoxy- 2-naphthyl)prop-2-en-1-one
}

The geometric parameters of the title molecule, $\mathrm{C}_{20} \mathrm{H}_{14} \mathrm{Cl}_{2} \mathrm{O}_{2}$, are in the normal ranges. The central $\mathrm{C}=\mathrm{C}$ double bond is trans configured and the two $\mathrm{C}$ atoms of this bond are slightly twisted out of the plane of the naphthyl group by 4.0 (3) $)^{\circ}$ The dihedral angle between the benzene ring and the naphthalene ring system is $44.94(4)^{\circ}$. The crystal packing is stabilized by $\mathrm{C}-\mathrm{H} \cdots \mathrm{O}$ and $\mathrm{C}-\mathrm{H} \cdots \mathrm{Cl}$ contacts.

\section{Comment}

Reviews on the bioactivities of various chalcones have been reported (Dimmock et al., 1999; Go et al., 2005). Recently, it has been noted that, among many organic compounds reported for their second harmonic generation, chalcone derivatives are known for their excellent blue light transmittance and good crystallizability (Fichou et al., 1988; Goto et al., 1991; Uchida et al., 1998; Zhao et al., 2000; Sarojini et al., 2006). The crystal structures of 3-(4-chlorophenyl)-1-(2-naphthyl)prop-2-enone (Shanmuga Sundara Raj et al., 1997), 1-(2naphthalenyl)-3-(3-nitrophenyl)-2-propen-1-one (Shanmuga Sundara Raj et al., 1998), 3-(6-methoxy-2-naphthyl)-1-(2naphthyl)prop-2-en-1-one (Yathirajan, Sarojini, Bindya et al., 2006), 3-(6-methoxy-2-naphthyl)-1-(2-thienyl)prop-2-en-1one (Yathirajan, Narayana et al., 2006), 1-(2,4-dichloro5-fluorophenyl)-3-(3,4-dimethoxyphenyl)prop-2-en-1-one (Yathirajan, Sarojini, Narayana et al., 2006) and (2E)-1-(2,4dichlorophenyl)-3-[4-(methylsulfanyl) phenyl]prop-2-en-1one (Butcher et al., 2007) have previously been reported. In continuation of our broad programme on chalcones, the present paper reports the crystal structure of a newly synthesized chalcone.<smiles>O=Cc1ccc(Cl)cc1Cl</smiles><smiles>COc1ccc2cc(C=O)ccc2c1</smiles>

$40 \% \mathrm{KOH}, \mathrm{MeOH}$
Received 19 December 2006 Accepted 20 December 2006

(C) 2007 International Union of Crystallography<smiles>CC(C)COC(C)O</smiles><smiles>COc1ccc2cc(/C=C/C(=O)c3ccc(Cl)cc3Cl)ccc2c1</smiles>

All rights reserved 
The molecular structure of (I) is shown in Fig. 1. Bond lengths and angles can be regarded as normal (Allen et al., 1987). The carbonyl group is twisted by $35.40(19)^{\circ}$ out of the plane of the dichlorophenyl ring. The torsion angle between the carbonyl group and the $\mathrm{C}$ atoms of the double bond is $-10.9(2)^{\circ}$. The torsion angle between the $\mathrm{C}$ atoms of the $\mathrm{C}=\mathrm{C}$ double bond and the plane of the adjacent naphthyl group $(\mathrm{C} 2-\mathrm{C} 3-\mathrm{C} 21-\mathrm{C} 22)$ is $4.0(3)^{\circ}$. The two aromatic groups are not coplanar [dihedral angle $=44.94(4)^{\circ}$ ]. The crystal packing is characterized by non-classical $\mathrm{C}-\mathrm{H} \cdots \mathrm{O}$ and $\mathrm{C}-\mathrm{H} \cdots \mathrm{Cl}$ hydrogen bonds (Table 1 ).

\section{Experimental}

To a thoroughly stirred solution of 2,4-dichloroacetophenone $(1.89 \mathrm{~g}$, $0.01 \mathrm{~mol})$ and 6-methoxy-2-naphthaldehyde (1.86 g, $0.01 \mathrm{~mol})$ in methanol ( $30 \mathrm{ml}), 40 \% \mathrm{KOH}$ solution $(5 \mathrm{ml})$ was added (see scheme). The mixture was stirred overnight and filtered. The product obtained was recrytallized from an acetone-toluene (1:1) mixture (m.p. 429$431 \mathrm{~K}$ ). Analysis for $\mathrm{C}_{20} \mathrm{H}_{14} \mathrm{Cl}_{2} \mathrm{O}_{2}$ : found (calculated): C 67.20 (67.24), H 3.86 (3.95)\%.

\section{Crystal data \\ $\mathrm{C}_{20} \mathrm{H}_{14} \mathrm{Cl}_{2} \mathrm{O}_{2}$ \\ $M_{r}=357.21$ \\ Monoclinic, $P 2_{1} / c$ \\ $a=16.8724(7) \AA$ \\ $b=13.4610(7) \AA$ \\ $c=7.4932(3) \AA$ \\ $\beta=102.778(3)^{\circ}$ \\ $V=1659.70(13) \AA^{3}$}

\section{Data collection}

Stoe IPDS-II two-circle diffractometer

$\omega$ scans

Absorption correction: multi-scan (MULABS; Spek, 2003; Blessing, 1995)

$T_{\min }=0.866, T_{\max }=0.873$

\section{Refinement}

Refinement on $F^{2}$

$R\left[F^{2}>2 \sigma\left(F^{2}\right)\right]=0.034$

$w R\left(F^{2}\right)=0.090$

$S=1.08$

3143 reflections

219 parameters

$\mathrm{H}$-atom parameters constrained

$$
\begin{aligned}
& w=1 /\left[\sigma^{2}\left(F_{\mathrm{o}}^{2}\right)+(0.041 P)^{2}\right. \\
& +0.8551 P] \\
& \text { where } P=\left(F_{\mathrm{o}}{ }^{2}+2 F_{\mathrm{c}}{ }^{2}\right) / 3 \\
& (\Delta / \sigma)_{\max }<0.001 \\
& \Delta \rho_{\max }=0.57 \mathrm{e}^{-3} \\
& \Delta \rho_{\min }=-0.21 \mathrm{e}^{-3} \\
& \text { Extinction correction: SHELXL97 } \\
& \text { Extinction coefficient: } 0.0156 \text { (15) }
\end{aligned}
$$

Table 1

Hydrogen-bond geometry $\left(\AA{ }^{\circ}\right)$.

\begin{tabular}{lllll}
\hline$D-\mathrm{H} \cdots A$ & $D-\mathrm{H}$ & $\mathrm{H} \cdots A$ & $D \cdots A$ & $D-\mathrm{H} \cdots A$ \\
\hline $\mathrm{C} 2-\mathrm{H} 2 \cdots \mathrm{O} 1^{\mathrm{i}}$ & 0.95 & 2.59 & $3.1367(19)$ & 117 \\
$\mathrm{C} 13-\mathrm{H} 13 \cdots \mathrm{O} 1^{\mathrm{ii}}$ & 0.95 & 2.59 & $3.535(2)$ & 174 \\
$\mathrm{C} 16-\mathrm{H} 16 \cdots \mathrm{Cl} 1^{\mathrm{iii}}$ & 0.95 & 2.83 & $3.5381(16)$ & 132 \\
\hline
\end{tabular}

Symmetry codes: (i) $x,-y+\frac{1}{2}, z-\frac{1}{2}$; (ii) $-x, y+\frac{1}{2},-z+\frac{1}{2}$; (iii) $-x, y-\frac{1}{2},-z+\frac{1}{2}$.

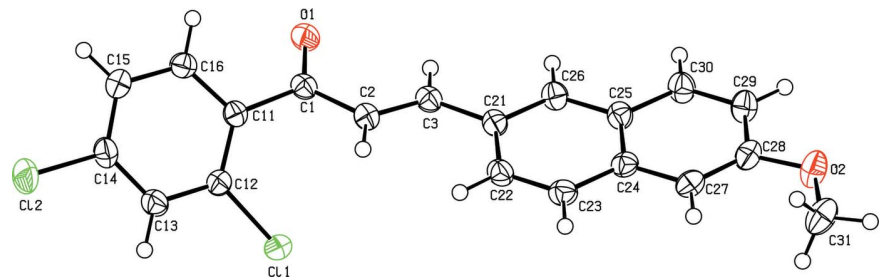

Figure 1

The molecular structure of (I), with displacement ellipsoids drawn at the $50 \%$ probability level.

$\mathrm{H}$ atoms were found in a difference map, but they were refined using a riding model with $\mathrm{C}-\mathrm{H}=0.95 \AA$ for $\mathrm{C}_{\text {aromatic }}$ and $\mathrm{C}-\mathrm{H}=$ $0.98 \AA$ for $\mathrm{C}_{\text {methyl }} . U_{\text {iso }}(\mathrm{H})$ was set to $1.2 U_{\text {eq }}(\mathrm{C})$, or $1.5 U_{\text {eq }}\left(\mathrm{C}_{\text {methyl }}\right)$. The methyl group was allowed to rotate but not to tip.

Data collection: $X$-AREA (Stoe \& Cie, 2001); cell refinement: $X$-AREA; data reduction: $X$-AREA; program(s) used to solve structure: SHELXS97 (Sheldrick, 1997); program(s) used to refine structure: SHELXL97 (Sheldrick, 1997); molecular graphics: PLATON (Spek, 2003); software used to prepare material for publication: SHELXL97 and PLATON.

ANM thanks the University of Mysore for permission to carry out the research work.

\section{References}

Allen, F. H., Kennard, O., Watson, D. G., Brammer, L., Orpen, A. G. \& Taylor, R. (1987). J. Chem. Soc. Perkin Trans. 2, pp. S1-19.

Blessing, R. H. (1995). Acta Cryst. A51, 33-38.

Butcher, R. J., Yathirajan, H. S., Narayana, B., Mithun, A. \& Sarojini, B. K. (2007). Acta Cryst. E63, o30-o32.

Dimmock, J. R., Elias, D. W., Beazely, M. A. \& Kandepu, N. M. (1999). Curr. Med. Chem. 6, 1125-1149.

Fichou, D., Watanabe, T., Takeda, T., Miyata, S., Goto, Y. \& Nakayama, M. (1988). Jpn J. Appl. Phys. 27, 429-430.

Go, M. L., Wu, X. \& Liu, X. L. (2005). Curr. Med. Chem. 12, 483-499.

Goto, Y., Hayashi, A., Kimura, Y. \& Nakayama, M. (1991). J. Cryst. Growth, 108, 688-698.

Sarojini, B. K., Narayana, B., Ashalatha, B. V., Indira, J. \& Lobo, K. J. (2006). J. Cryst. Growth, 295, 54-59.

Shanmuga Sundara Raj, S., Ponnuswamy, M. N., Shanmugam, G. \& Nanjundan, S. (1997). Acta Cryst. C53, 917-918.

Shanmuga Sundara Raj, S., Ponnuswamy, M. N., Shanmugam, G. \& Nanjundan, S. (1998). Acta Cryst. C54, 541-542.

Sheldrick, G. M. (1997). SHELXS97 and SHELXL97. University of Göttingen, Germany.

Spek, A. L. (2003). J. Appl. Cryst. 36, 7-13.

Stoe \& Cie (2001). X-AREA. Stoe \& Cie, Darmstadt, Germany.

Uchida, T., Kozawa, K., Sakai, T., Aoki, M., Yoguchi, H., Abduryim, A. \& Watanabe, Y. (1998). Mol. Cryst. Liq. Cryst. 315, 135-140.

Yathirajan, H. S., Narayana, B., Ashalatha, B., Sarojini, B. K. \& Bolte, M. (2006). Acta Cryst. E62, o4440-o4441.

Yathirajan, H. S., Sarojini, B. K., Bindya, S., Narayana, B. \& Bolte, M. (2006). Acta Cryst. E62, o4046-o4047.

Yathirajan, H. S., Sarojini, B. K., Narayana, B., Bindya, S. \& Bolte, M. (2006). Acta Cryst. E62, o3631-03632.

Zhao, B., Lu, W.-Q., Zhou, Z.-H. \& Wu, Y. (2000). J. Mater. Chem. 10, 15131517. 